\title{
¿Tirando viejos por la ventana? Militancia juvenil y gestión estatal en el Movimiento Evita de Argentina $(2005-2015)^{*}$
}

\author{
Pulling the old out the window? Youth militancy and state \\ management in the Evita Movement of Argentina (2005-2015) \\ Jogando idosos pela janela? Militância juvenil e gestão do Estado no \\ Movimento Evita da Argentina (2005-2015)
}

Recibido el 12 de junio de 2017. Aceptado el 1 de marzo de 2018

Francisco Longa*

Argentina

\section{Resumen}

Para citar este artículo:

Longa, Francisco (diciembre,

2018). ¿Tirando viejos por la ventana? Militancia juvenil y gestión estatal en el Movimiento Evita de Argentina (2005-2015). Ánfora, 25(45), 197-218. DOI: https://doi.org/10.30854/anf. v25.45.2018.XXX Universidad Autónoma de Manizales. ISSN 0121-6538.
Objetivos: analizar la experiencia del Movimiento Evita de Argentina para comprender los sentidos que en el movimiento se construyen en torno a la noción de 'juventud', y los accesos y restricciones de dicha juventud a las estructuras del movimiento. Además, se analiza la mirada de los jóvenes del movimiento en torno a la ocupación de cargos de gestión estatal y la apropiación de la juventud respecto del 'kirchnerismo'. Metodología: a partir de técnicas de investigación cualitativas, que priorizaron la realización de entrevistas en profundidad y las visitas de campo, se estudiaron las categorías de 'vitalidad' y 'pureza' y los accesos a instancias de toma de decisión para la juventud en el

\footnotetext{
* Artículo producido en el marco de una beca del Consejo Nacional de Investigaciones Científicas y Técnicas (CONICET) de Argentina, iniciada en marzo de 2012. Título de la tesis: “¿Entre la autonomía y la disputa institucional? El dilema de los movimientos sociales ante el Estado. Los casos del Frente Popular Darío Santillán y el Movimiento Evita (Argentina, 2003-2015)", Facultad de Ciencias Sociales, Universidad de Buenos Aires (UBA). La tesis fue defendida y aprobada en octubre de 2016. El presente artículo se desprende también del trabajo de investigación a partir de una beca posdoctoral del CONICET de Argentina, con plazo entre 2017 y 2019. El autor agradece los valiosos comentarios de los evaluadores anónimos.

** Doctor en Ciencias Sociales. Magíster en Investigación en Ciencias Sociales. Licenciado en Ciencia Política. Investigador becario del CONICET con sede en el Instituto de Investigaciones en Humanidades y Ciencias Sociales de la Universidad Nacional de La Plata. Correo: francisco_longa@yahoo.com.ar.
} 
movimiento. Resultados: mientras que existe un consenso en torno a que los jóvenes ganen espacios en la estructura del movimiento, aparecen también restricciones en su acceso a las instancias de toma de decisión. Esto es contrapesado con el importante porcentaje de cargos legislativos ocupados por la juventud. Además, la juventud del movimiento muestra una fuerte apropiación respecto del 'kirchnerismo'. Conclusiones: el escenario muestra accesos y restricciones de los jóvenes en las estructuras y espacios del Movimiento Evita. Además, la adhesión de los jóvenes hacia el kirchnerismo no tuvo tanto que ver con el acceso a cargos estatales, sino con la valoración positiva de las políticas públicas impulsadas entre 2003 y 2015 por el gobierno nacional.

Palabras clave: Movimientos sociales; Estado; Juventud; Argentina.

\section{Abstract}

Objective: to analyze the experience of the Evita Movement in Argentina in order to understand the meanings that the movement builds around the notion of 'youth', and the access and restrictions of such youth to the structures of the movement. In addition, it analyzes the young people's view of the movement with regard to the occupation of positions in state management and the appropriation of the youth with respect to 'Kirchnerism. Methodology: based on qualitative research techniques, which prioritized the conduct of in-depth interviews and field visits, the categories of 'vitality' and 'purity' were studied, as well as access to decision-making bodies for young people in the movement. Results: while there is a consensus that young people gain space in the movement's structure, there are also restrictions on their access to decisionmaking bodies. This is counterbalanced by the significant percentage of legislative positions held by young people. In addition, the young people in the movement show a strong appropriation regarding 'Kirchnerism'. Conclusions: the scenario shows access and restrictions of young people in the structures and spaces of the Evita Movement. In addition, the adhesion of young people to Kirchnerism had not so much to do with access to state positions, but with the positive assessment of public policies promoted between 2003 and 2015 by the national government.

Keywords: Social movements; State; Youth; Argentina.

\section{Resumo}

Objetivo: analisar a experiência do Movimento Evita na Argentina para compreender os sentidos que o movimento constrói em torno da noção de 'juventude' e os acessos 
e restrições dessa juventude às estruturas do movimento. Além disso, analisa-se o olhar dos jovens do movimento em torno da ocupação de posições de gestão estatal e a apropriação da juventude em relação ao 'Kirchnerismo'. Metodologia: com base em técnicas de pesquisa qualitativa, que priorizaram a realização de entrevistas em profundidade e visitas de campo, foram estudadas as categorias de 'vitalidade' e 'pureza', bem como os acessos a órgãos de tomada de decisões para os jovens do movimento Resultados: enquanto existe um consenso de que os jovens ganham espaço na estrutura do movimento, aparecem restrições em seu acesso aos órgãos decisórios. Isso é contrabalançado pela porcentagem significativa de posições legislativas ocupadas por jovens. Além disso, a juventude do movimento mostra uma forte apropriação em relação ao ' Kirchnerismo'. Conclusões: o cenário mostra acessos e restrições dos jovens nas estruturas e espaços do Movimento Evita. Ainda mais, a adesão de jovens ao kirchnerismo não teve tanto a ver com o acesso aos cargos estaduais, mas com a avaliação positiva das políticas públicas promovidas entre 2003 e 2015 pelo governo nacional.

Palavras - chave: Movimentos sociais; Estado; Juventude; Argentina. 


\section{Introducción}

La participación de los movimientos sociales en el Estado y en los gobiernos ha sido un nudo teórico relevante para la academia contemporánea. Esto, porque a diferencia de otros formatos organizativos, como los partidos políticos o los movimientos de liberación nacional, el surgimiento de los llamados movimientos sociales en Europa y Estados Unidos -durante las décadas de 1960 y 1970- comportó una novedad, en la medida en que la mayor parte de estos movimientos reivindicaba la ampliación de derechos para determinados colectivos sociales, pero se mostraba renuente a la toma del poder estatal.

En función de lo anterior, en la academia se fue configurando un 'dilema' teórico que planteaba que los movimientos sociales que viraban en sus orientaciones y finalmente asumían la disputa estatal perdían sus perfiles emancipatorios, cayendo en las dinámicas burocratizantes del Estado. No obstante, aquellos movimientos que no disputaran la arena político institucional podían pasar a la intrascendencia cuando no a la disolución, perdiendo capacidad de irradiación en la sociedad; algunos de los principales teóricos que dieron cuenta de este 'dilema' fueron Roberto Unger (1987) y Gerardo Munck (1995). Por su parte, Goldstone (2003) planteó que estos movimientos constituyen un elemento central del escenario político contemporáneo y que establecen una relación compleja -de conflictividad y complementariedad- respecto de la política institucional.

En Argentina el 'dilema' teórico y el rol de los movimientos sociales, emergió fuertemente durante el ciclo de luchas instituyentes de finales del siglo XX y principios del siglo XXI, en el marco de la proliferación de un conjunto de movimientos sociales autogestivos como asambleas barriales, fábricas recuperadas y movimientos de trabajadores desocupados, entre otros. Estas experiencias se dedicaron a la construcción de base en los barrios pobres y/o en los lugares de trabajo y rechazaron la disputa institucional (Zibechi, 1999). Sin embargo, desde la asunción del presidente Néstor Kirchner en 2003, los contornos de este debate cambiaron sustancialmente, experimentándose un verdadero cambio de época en el país (Svampa, 2008).

En ese nuevo escenario, muchos movimientos sociales pasaron a ocupar cargos de gestión estatal y a adherir a los gobiernos de Néstor Kirchner primero (entre 2003 y 2007) y de Cristina Fernández de Kirchner luego (2007-2011 y 2011-2015). Por otra parte, más precisamente desde el segundo gobierno de Cristina Fernández de Kirchner, la 'juventud' como categoría cobró inusitada fuerza (Vázquez, 2013) y jugó un rol clave en las narrativas y discursos proferidos desde el Estado (Larrondo, 2013), principalmente en aquellos ligados al rol de la militancia en el proyecto político kirchnerista. 
El problema que abordan las líneas que siguen es cómo se configura el imaginario acerca de la 'juventud' en el Movimiento Evita y qué accesos y restricciones se observan para los/as jóvenes en las estructuras del movimiento. Además, el objetivo del artículo apunta a conocer las valoraciones y los modos de apropiación de la juventud del movimiento respecto del proyecto político 'kirchnerista' y las tensiones en esta apropiación respecto del acceso a los cargos gubernamentales.

\section{El Movimiento Evita y el kirchnerismo}

"Eso es lo que plantea la JP [Juventud Peronista ] Evita hoy en día (...) los compañeros lo aceptaron para bien y ya muchos están diciendo que el espacio lo tienen que empezar a conducir los pibes (...) parte de los compañeros mucho más grandes están aceptando de buena manera. Bueno en un principio pensaron como que - 'me están mojando la oreja', pero pudieron entender y con risa nos dijeron: - 'bueno está todo bien, pero no tiren todos los días un viejo por la ventana"” (Lucas, militante de la JP Evita, prov. de Buenos Aires)

El Movimiento Evita es un movimiento social conformado entre finales de 2004 y principios de 2005 en Argentina, que se estructura a través de frentes de masas y de secretarías. Los frentes de masas más dinámicos y numerosos del Movimiento Evita son el de juventud y el de economía popular. La rama de la economía popular está integrada principalmente por trabajadores/as desocupados/as, que se nuclean a partir de subsidios estatales. La principal base social del movimiento la constituyen vecinos y vecinas de las barriadas populares de la periferia de la Ciudad de Buenos Aires, quienes realizan tareas comunitarias a cambio de los subsidios estatales en los cuales son inscriptos/as por participar del movimiento. Uno de los principales formatos de organización de esta población son las cooperativas de trabajo, que en general se dedican a la limpieza de las calles de sus propios barrios, al saneamiento de arroyos y zanjas o que se orientan al trabajo productivo en carpinterías, herrerías o talleres de costura.

El caso de estudio que se presenta, se organiza internamente de un modo en el que la 'juventud' tiene un canal orgánico: la Juventud Peronista Evita (JP Evita). La JP Evita es una de las ramas más dinámicas y numerosas del movimiento y se dedica centralmente a desarrollar trabajo barrial en territorios populares. En estos espacios el movimiento brinda asistencia alimentaria a familias pobres, realiza talleres educativos, de formación política, artísticos y de capacitación laboral. Por citar el caso del municipio de San Fernando, la JP Evita 
está conformada por un núcleo militante de cerca de 80 jóvenes de entre 15 y 25 años y tiene una capacidad de movilización de entre 400 y 600 personas. A la vez, esta rama articula fuertemente con el frente de la economía popular, donde se organizan las cooperativas del movimiento, sus espacios de comercialización, producción y de lucha por derechos laborales.

Los antecedentes organizativos del Movimiento Evita reenvían a una serie de agrupamientos que se formaron durante la década de 1990 en las barriadas pobres del 'gran Buenos Aires'. Esta región, la más densamente poblada del país, reúne cerca de 14 millones de habitantes y presenta altos índices de población con necesidades básicas insatisfechas (Zarazaga, 2017). Estas organizaciones aglutinaban a desocupados y desocupadas y se organizaban centralmente en Movimientos de Trabajadores Desocupados (MTD).

Desde 2003 el Movimiento de Trabajadores Desocupados Evita (MTD Evita) y otros grupos como el Peronismo que Resiste, el Movimiento Patriótico Malón, el Movimiento Popular 20 de diciembre, Descamisados y el Movimiento la Patria Vencerá, comenzaron un recorrido de coordinaciones y articulaciones que los llevó a conformar el espacio Patria o Muerte. Si bien este espacio se reconocía como nacional-popular y reivindicaba las gestiones presidenciales de Juan Domingo Perón (1946-1955 y 1973-1974), durante esos años de lucha se volcó a la construcción de organizaciones de base en las barriadas pobres, mientras que se mostró crítico de la participación electoral. Como ejemplo, frente a las elecciones presidenciales de 2003 donde fue electo Néstor Kirchner, declararon: "la partidocracia corrupta sigue sin acusar recibo. Se prepara, como si nada, para montar una nueva farsa electoral"1.

Pero luego de la asunción a la Presidencia de la Nación de Néstor Kirchner esto cambió. El nuevo presidente implementó una serie de medidas de gobierno progresistas, como el impulso de los juicios a los militares responsables de la última dictadura militar o el alineamiento internacional con el bloque de gobiernos antineoliberales de América Latina como los de Hugo Chávez en Venezuela y Evo Morales en Bolivia. Esto se leyó como un agenda de demandas de los sectores populares (Cheresky, 2004) y hasta llevó a que se denomine a Néstor Kirchner como presidente de centroizquierda (Alcántara, 2008).

\footnotetext{
1 Luego proponía: "hacemos un Ilamado a auto convocarnos todos los sectores dispuestos a movilizarnos y construir una gran contra campaña que a lo largo y ancho del país, desde ahora y hasta el día de las elecciones, les recuerde lo que ya parecen haber olvidado: que tienen que irse todos". El documento se tituló Contra la farsa electoral, por otro 20 de diciembre: que se vayan todos, y llevó las firmas de las organizaciones: Patria o Muerte, Movimiento Barrios de Pie - Movimiento Independiente de Jubilados y Desocupados (MIJD) - Coordinadora de Trabajadores Desocupados (CTD) Aníbal Verón - Frente Barrial 19 de Diciembre. Para esta fecha, el MTD Evita formaba parte de Patria o Muerte. Más información en http://www.lafogata.org.cn2.toservers.com/003arg/arg3/ar_farsa.htm
} 
En este nuevo contexto, algunas de las agrupaciones de Patria o Muerte formaron el Frente de Organizaciones Populares (FOP) y pasaron a apoyar al presidente Kirchner. Entre finales de 2004 y principios de 2005 este espacio cambió de nombre nuevamente, incorporó nuevos grupos y se constituyó oficialmente como Movimiento Evita (Natalucci, 2012). Desde sus inicios el Movimiento Evita se sumó a la gestión de gobierno de la Provincia de Buenos Aires bajo la gobernación de Felipe Solá, aliado de Néstor Kirchner. Según Martín Cortés, el paso del MTD Evita al Movimiento Evita no puede pensarse por fuera de la cuestión del Estado: "el salto hacia la intervención política (esto es, de MTD Evita a Movimiento Evita) está pensado para incidir en el direccionamiento de las políticas de Estado y construir herramientas de organización para el campo popular" (Cortés, 2010, p. 108). No obstante, lejos de abandonar la construcción social en los barrios populares, el movimiento comenzó a hilvanar una trayectoria en donde participación social y gestión estatal estuvieron íntimamente relacionadas (Longa, 2017).

\section{Metodología}

El presente artículo se posiciona desde un enfoque cualitativo, que se valió de una estrategia metodológica multimétodo. Las principales técnicas de construcción y de recolección de datos fueron las entrevistas en profundidad y las visitas y observaciones de campo. Estas técnicas responden a las necesidades que plantean los objetivos de investigación expuestos más arriba.

Las entrevistas permiten acceder a las miradas y representaciones que tienen los y las militantes acerca de las nociones asociadas a la idea de 'juventud' en el movimiento. También permiten indagar en los vínculos de la juventud con la gestión estatal. Para las entrevistas se confeccionó una muestra en forma estratégica, es decir que la mayoría de los/as entrevistados/as fue seleccionada a partir del criterio del autor, tal como corresponde a esta modalidad de muestreo no probabilístico (Sabino, 2000; Vieytes, 2004).

Los nombres de los y las entrevistadas que aparecen citados en este trabajo han sido modificados para preservar sus identidades. Son citados con seudónimo en el cuerpo del texto entre paréntesis, acompañados de las iniciales que refieren al movimiento, verbi gratia, Lucas -ME-. Solamente en los casos en los cuales se entrevistó o se cita a funcionarios/as, legisladores/as o referentes con un alto reconocimiento público se consignó su nombre verdadero. Cuando se trata de éstos últimos, se detalla también el apellido y, en la primera aparición, se inserta una nota que describe su cargo público o su condición de referente o dirigente; 
esta forma de organizar los testimonios se recuperó de trabajos con movimientos sociales contemporáneos como el de Quirós (2008).

Respecto de las observaciones, esta técnica se articula con el objetivo de comprender qué lugar ocupa la juventud en la estructura orgánica del movimiento. En las observaciones se asistió a talleres, movilizaciones, actos de campaña, actividades barriales y jornadas de trabajo de las cooperativas del movimiento elegido. Esto permitió contemplar el peso específico que tienen los y las jóvenes en general y las actividades de la JP Evita en particular, en la vida cotidiana del movimiento. Durante dichas observaciones se confeccionaron diferentes tipos de notas que se corresponden con los presentados por Valles (1997), es decir: notas condensadas, citas textuales durante las observaciones y notas expandidas: ampliación en detalle de las notas condensadas después de la observación.

\section{Resultados}

\section{"La relación con los pibes es muy buena, si no te tiran por el balcón" (Victor, militante adulto del Movimiento Evita, Prov. de Buenos Aires).}

En el apartado que sigue se exponen en primer lugar los imaginarios de los y las militantes del Movimiento Evita en relación con la noción de 'juventud' y los aspectos positivos y negativos que contienen dichos imaginarios. En segundo lugar, se describen los accesos y las restricciones que la juventud tiene en la estructura orgánica del movimiento, principalmente en los espacios de toma de decisión. Más adelante, se muestra que las políticas públicas ligadas a la juventud fueron decisivas para la apropiación por parte de la militancia del Evita del proyecto kirchnerista. Finalmente, se desarrolla el lugar de los y las jóvenes en los cargos ejecutivos y/o legislativos a los que el movimiento accedió durante los gobiernos kirchneristas.

En el campo de las ciencias sociales la perspectiva biologicista y/o escencialista para definir a la juventud, que fue expresada por trabajos como los de Sherif y Sherif (1975), se encuentra actualmente superada. Los enfoques más aceptados reconocen en cambio que tanto 'vejez' como 'juventud' no están dadas, "sino que se construyen socialmente en la lucha entre jóvenes y viejos" (Bourdieu, 1990, p. 164). Por ello, la conformación de la juventud debe entenderse "en el contexto de las luchas sociales por la reproducción, luchas entre grupos sociales por el control del acceso a las distintas posiciones en la sociedad" (Martín, 1998, p. 36). 
Como fue dicho, en el Movimiento Evita existe un canal orgánico específico para la juventud: la JP Evita. En las extensas visitas de campo y entrevistas realizadas con militantes de base, con cuadros medios y con referentes del Evita, se pudo conocer en profundidad el trabajo de la JP Evita, principalmente en los municipios de San Fernando, Vicente López y Avellaneda, todo ellos en la provincia de Buenos Aires y la Capital Federal de la Argentina.

Dicha investigación de campo mostró el importante dinamismo y volumen de la militancia de la JP Evita. Estos datos confirman el testimonio de uno de sus referentes, para quien la juventud: "el frente más grande que tiene el Movimiento Evita acá en San Fernando, organizado. Hay muchos compañeros del Movimiento Evita, pero lo más organizado y lo más nítido es la JP Evita” (Lucas -ME-). Más allá del aspecto cuantitativo, sin embargo, lo que se observa en relación con el lugar de la juventud en el movimiento es que éste se constituye en un continuo péndulo de accesos y restricciones respecto del lugar de los adultos. Esto se observa tanto en los testimonios como en algunas estructuras.

Respecto de los testimonios, es innegable que en el Movimiento Evita tanto para los jóvenes como para los adultos la 'juventud' como concepto viene aparejada de una serie de virtudes, como por ejemplo la de oficiar de base principal del movimiento: "el andamiaje y me atrevo a decirlo, de todo lo que es el Movimiento Evita, es la JP. Los mejores cuadros que tiene la organización están en la juventud” (Gerardo -ME-). En la medida en que Gerardo es un militante adulto del Evita, su testimonio concuerda con lo planteado por Melina Vázquez (2013), quien sostuvo que la noción de juventud en las militancias actuales no es el resultado de una disputa intergeneracional, sino en muchos casos producto de una construcción de sentido ejercida tanto por adultos como por jóvenes, quienes confluyen en destacar determinadas virtudes que caracterizarían a lo juvenil: "dirigentes y funcionarios adultos hablan a -y acerca de- los jóvenes exaltando la importancia de la participación, el compromiso y la militancia” (Vázquez, 2013, p. 5).

El testimonio de Silvia, militante adulta del municipio de Vicente López lo reafirma; para ella, los jóvenes representan: "todo; la energía, la voluntad. Sin los pibes no hay nada. Si no hay pibes esto se muere. En la frescura, en la militancia, en afichar, en pintar, están continuamente con eso" (Silvia-ME-). Esta narrativa coincidiría con las ideas de 'vitalidad' y 'frescura' de la juventud, lo que lleva como contrapartida a relacionar a los adultos con una práctica política turbia, plagada de internismos y de negociaciones espurias. En tal sentido, para Lucas los adultos: "se quedan mucho más en la rosca²; y yo creo que esa es la diferencia que tienen los pibes, por eso nosotros aglutinamos la cantidad de compañeros

2 En el lenguaje nativo de la militancia en Argentina, la rosca refiere a la negociación, muchas veces solapada o espuria, entre diversos dirigentes políticos. 
que aglutinamos, y vamos a las marchas con todo el fervor y cada día somos más (Lucas -ME-).

Es por todos estos valores renovadores, que se impone el desplazamiento de los adultos y la necesidad de 'dar lugar' a la juventud (Vázquez, 2013). Eso puede advertirse en otro testimonio de Gerardo: "vamos quedando viejos, y está bueno digo, porque si uno a los cuarenta o cuarenta y pico ya en el espacio estás grande, significa que los pibes vienen creciendo y corriendo" (Gerardo -ME-). Sin embargo, Gerardo reconoce que existe un porcentaje de adultos que no acepta de buena gana el avance de la juventud en la organización: "la mayoría festeja este avance, este trasvasamiento generacional que se va dando; no puedo decir que todos, pero yo calcularía que un 80\% está festejando” (Gerardo-ME-). Tal vez al otro $20 \%$ se refiere Lucas cuando sostiene que: "los viejos lo primero que te dicen es -'eh vos ¿qué me querés enseñar política a mí? Si cuando vos estabas en los huevos de tu padre yo ya estaba haciendo política”.

No obstante, el propio Lucas coincide con Gerardo en que esos posicionamientos son minoritarios o bien que eran más comunes años atrás: “están aceptando que (...) se tienen que jubilar y que tienen que dejar el espacio a otros compañeros y eso me pone contento. Por eso te digo que se van resignando y van aceptando que la conducción tiene que ser la nueva generación” (Lucas -ME-). En este caso, no obstante, el 'dar lugar a la juventud' no estaría tan ligado al convencimiento acerca del carácter renovador, puro y fresco de las nuevas camadas, sino a la resignación acerca de lo que los adultos ya no pueden hacer. Es por esto que el 'dar lugar a los jóvenes' no se configura solamente a partir de las virtudes de la juventud, sino también desde las propias limitaciones de los adultos.

Además, estas resistencias y resignaciones de los adultos responden a una mirada algo despreciativa, que advierte carencias en los jóvenes. Las carencias más recurrentes refieren a la ingenuidad y a la falta de capacitación: "los pibes están muy bien encuadrados, se capacitan, les falta por ahí más; yo siempre les planteo que para el 2019, dentro del marco vamos a decir de las elecciones, yo voy a tener que militar para ellos" (Víctor -ME-). El período de tiempo por venir sería el encargado de subsanar esa 'falta' que los adultos identifican en los jóvenes. En el caso de Silvia, esta falta genera una juventud con cierta ingenuidad, lo cual advierte cuando se piensa la toma de decisiones en el movimiento:

"Es vertical, como siempre. Uno se puede hacer el boludo, pero en realidad la toma de decisiones no sale de nosotros. Para mi es así. Si bien lo hablamos, lo charlamos. Yo lo veo de mi lugar de grande eh! Los pibes a lo mejor te opinan totalmente distinto, te dicen -'esto es amplio, se discute, se debate'. Pero una que es más grande ve que hay cosas que ya están decididas” (Silvia-ME-). 
Como se observa, su capacidad 'adulta' de zanjar la ingenuidad le permite identificar que el hecho de que haya instancias de debate colectivo no implica que la toma de decisiones sea horizontal, como sería entendido desde la ingenuidad de 'los pibes'. Aun así, esto es visto como una carencia transicional que con el pasaje a la madurez puede subsanarse: "yo creo que hay que acompañar con la experiencia, con lo que ha pasado; acompañar, empujar hacia arriba a los pibes, a las pibas para que organicen, e ir corriéndote" (Silvia -ME-).

Además de los testimonios es interesante dar cuenta del espacio concreto que los jóvenes ocupan en las estructuras del movimiento, sobre todo en lo que refiere a la toma de decisiones. Precisamente un militante adulto advierte que los jóvenes no tienen en el movimiento todo el espacio que merecen: "yo creo que hay una resistencia, si bien se le da mucha participación. Porque el Movimiento Evita más que nada está conformado por pibes. Pero ¿cuántos hay en la Mesa Nacional?” (Víctor -ME-). Víctor se refiere a la Mesa Ejecutiva Nacional: la máxima instancia de toma de decisión del Movimiento Evita. Hoy en día está conformada por diez personas, de las cuales tan sólo una tiene menos de 35 años. El porcentaje indica entonces que apenas un $10 \%$ de los lugares de dicha mesa está ocupado por jóvenes, lo cual confirma lo sostenido por Víctor acerca de la incongruencia entre el volumen mayoritario de militancia de la JP Evita y su escasa representación en el espacio más importante de toma de decisiones.

Claro que el movimiento tiene una compleja y profunda estructura de toma de decisiones, y que es probable que los jóvenes tengan más presencia en instancias de menor rango, como en las mesas provinciales de decisión o en los espacios municipales. Precisamente, el trabajo de campo en un municipio de Buenos Aires permitió advertir que la escala local es más permeable a los/las jóvenes. En ese caso, la juventud se ganó allí un espacio de decisión pero luego de una verdadera compulsa de poder frente a los adultos:

"Los pibes, al ver que no teníamos una respuesta frente a eso y siendo mayoría también, siendo el frente más grande que tiene el Movimiento Evita acá (...) entonces decidimos, si hay cosas que funcionan mal y los compañeros más grandes no lo escuchan, parémonos todos en la mesa y vamos a decirles -'miren nosotros somos mayoría, o aceptan lo que dicen las bases o ¿a qué están jugando muchachos'? (Lucas -ME-).

Luego de dicho planteo, en el distrito se habilitaron canales para que los y las jóvenes tengan más participación en las decisiones. Este escenario local contrasta con lo visto para la Mesa Ejecutiva Nacional, la cual, tomando un criterio estrictamente biológico y cuantitativo daba cuenta de un acceso restringido de la juventud a las decisiones. Al momento, empero, de sopesar las restricciones o 
permeabilidades que tiene la juventud en el movimiento también debe tenerse en cuenta su presencia en los cargos estatales, a lo cual se dedica el próximo apartado.

\section{El Estado de la gestión}

El acceso a la gestión del Estado por el Movimiento Evita durante el kirchnerismo es otra de las dimensiones en donde se pueden observar restricciones y avances para la juventud. En primer lugar hay que aclarar que, en general, durante el kirchnerismo el Movimiento Evita profundizó su acceso al Estado como método complementario a su construcción social. Emilio Pérsico, el Secretario General del Movimiento Evita, sostiene que el rol del movimiento debe ser el de mediar entre la sociedad y el Estado, intentando que la estructura estatal resuelva los conflictos y las desigualdades en forma beneficiosa para los sectores populares. Esa perspectiva es la que según él caracterizó al peronismo como corriente política:

El conflicto hay que meterlo para adentro y darle solución. Eso es lo que hizo el peronismo centralmente. Asumir el conflicto que estaba en la calle, lo metían adentro del Estado y el Estado trataba de darle solución (...) y esta es la tarea del Estado y de la organización popular (Gómez y Massetti, 2009, p. 26).

En el plano conceptual, entonces, en el movimiento anida una valoración positiva acerca de las capacidades del Estado de mediar en el conflicto social en función de un beneficio para los sectores populares. Se ha dicho que desde 2005 el movimiento integró a alguno de sus militantes en cargos de gestión estatal, así también como en listas electorales. El nivel de institucionalización de los vínculos entre movimiento y Estado muestra un escenario donde la ocupación de puestos estatales es tal-desde empleados hasta cargos ejecutivos- que todo el andamiaje organizacional parece constituirse desde las posiciones ganadas en la estructura institucional.

De hecho, el movimiento experimentó un fuerte crecimiento desde la llegada de Pérsico al Ministerio de Desarrollo Social de la Nación en 2009, donde fue nombrado Subsecretario de Comercialización de la Economía Social de la Nación "con un presupuesto de 750 millones que [finalmente] sería de 9.000 millones” (Boyanovsky, 2010, p. 203). En el trabajo de campo se pudo observar cómo en la medida que el movimiento comenzó a desplegar operativos de programas estatales en los territorios multiplicó su presencia barrial. Esto ha llevado a Boyanovsky a afirmar que: "el Movimiento Evita parece ser el que mejor repite el esquema peronista de crecimiento a partir del poder institucional” (2010, p. 222). 
Pero en lo que refiere al sujeto juvenil del movimiento, aparece un aspecto de la construcción que marca distancia respecto de los recursos estatales. $\mathrm{Mu}$ chos de los militantes del movimiento se mantuvieron durante estos años al margen de los cargos estatales y continuaron trabajando en empleos formales en empresas o realizando trabajos en forma independiente. En un estudio sobre la gestión de cargos estatales de otra organización kirchnerista, Melina Vázquez resaltó que durante los últimos años asistimos a la estigmatización pública de "las agrupaciones juveniles kirchneristas, concretamente en relación con la idea de que se trata de una 'militancia rentada', en la cual el principal objetivo de sus miembros es obtener cargos en la gestión pública” (Vázquez, 2014, p. 82). Tal vez en función de rebatir la mirada estigmatizadora, es que los jóvenes de la JP Evita remarcan con especial énfasis que parte de sus ingresos provienen de la autogestión y que muchos de sus militantes no ocupan ni ocuparon cargos estatales durante el kirchnerismo:

"Hay compañeros que no están laburando dentro del Estado, que son los que también ponen la tarasca $a^{3}$ para mantener las actividades. Por ejemplo tenemos compañeros que laburan en Coto, son repositores, y cuando tienen un tiempito te dicen -'no, yo me pago el ferrite'. Viene el compañero que trabaja en Easy y te dice: -'no, yo voy a arreglar los parches de los instrumentos'. Viene otro compañero que trabaja en el tren o alguno que hace alguna changa y dice: -'che, yo dejo estos pesos'. Y está la compañera que también es costurera que te da una mano. Entre los compañeros también todo artesanalmente, las remeras las hacemos nosotros, las banderas las hacemos nosotros" (Lucas -ME-). ${ }^{4}$

Más allá de este testimonio, que bien podría representar una estrategia de presentación (Potter y Wetherell, 1987) del entrevistado en función de rebatir el estigma público sobre la ocupación de cargos estatales antes mencionado, lo cierto es que el trabajo de campo con observaciones directas en el territorio permitió constatar la veracidad del testimonio. En el trabajo de campo se observó una diversidad de actividades que cotidianamente el Movimiento Evita en general y la JP Evita en particular, desarrollan al margen de programas específicos de gobierno, incluso también deslindados de recursos estatales.

Los ejemplos de los roperos comunitarios en los barrios populares, que sirven para juntar dinero para financiar actividades, o el caso de la confección de

\footnotetext{
3 En el argot popular de Argentina, tarasca refiere al dinero.

4 Tanto Coto como Easy son grandes hipermercados privados. La mayoría de los entrevistados trabaja allí de repositores o cajeros, es decir en empleos para los cuales se demanda poca calificación y se otorgan bajas remuneraciones.
} 
banderas del movimiento en forma artesanal hechas por jóvenes de la JP Evita, o las innumerables actividades convocadas en el ámbito universitario por el Movimiento Universitario Evita, hablan de un conjunto importante de actividades poco institucionalizadas, en el sentido de que se pueden llevar a cabo sin la necesaria intervención de reconocimiento o de recursos provenientes del Estado.

Por otro lado, y más allá de los cargos de gestión ejecutivos, también es importante mencionar que la juventud del movimiento ha tenido un lugar importante en las listas a cargos legislativos en diversas instancias electorales. Tomando como ejemplo las elecciones de 2011, en las cuales se eligieron cargos ejecutivos y legislativos en todo el país, el Movimiento Evita logró colocar a una serie de militantes en las listas del Frente Para la Victoria (FpV), el partido de gobierno conducido por Cristina Fernández de Kirchner.

En la Provincia de Buenos Aires el Movimiento Evita colocó a cinco militantes compitiendo para diversos cargos $^{5}$. De esos cinco, dos eran menores de 30 años y tres mayores de 45 años; es decir que el 40\% de las candidaturas estuvieron por debajo de los 30 años. Hay que destacar, además, que todos/as los militantes de las listas lograron entrar a sus cargos, lo que muestra que no solamente la juventud participó de las listas electorales sino que lo hizo en puestos que les permitieron acceder a las bancadas. Más aún, para las elecciones de 2015 donde se renovaron todos los cargos de 2011, el Movimiento Evita solamente logró colocar a 1 de sus militantes en las listas del FpV: se trató de Leonardo Grosso, que para dicho año tenía apenas 31 años.

Además de estos cargos electorales provinciales, varios jóvenes del Evita ocuparon las listas para cargos legislativos municipales, como los casos de Federico Ugo y Joaquín Noya, ambos concejales de municipios del conurbano bonaerense. Todos ellos/as fueron electos teniendo menos de 40 años de edad. También es destacable el caso de Natalia Soria, militante del Movimiento Evita que con solo 36 años logró en 2011 acceder al cargo de intendenta del municipio de Valle Viejo, en la provincia de Catamarca.

Esta imagen de acceso a instancias electorales para la juventud contrasta claramente con las restricciones vistas para el caso de la toma de decisiones. Es evidente que ese $40 \%$ de los lugares en los puestos electorales bonaerenses del 2011, implica un acceso mucho más amplio que aquel 10\% de presencia juvenil de la Mesa Ejecutiva Nacional. Para seguir indagando en la relación que la juventud del Evita estableció con las estructuras estatales y con el proyecto político kirchnerista, el siguiente apartado focaliza en las iniciativas para la ju-

5 Los militantes disputaron cargos de diputados provinciales, diputados nacionales y de senadores provinciales. 
ventud impulsadas por la JP Evita y en las políticas públicas que desde el Poder Ejecutivo se impulsaron al respecto.

\section{Promover desde fuera y gestionar desde dentro}

Además de la inserción en cargos legislativos, también hay que destacar una serie de iniciativas relativas a la juventud que el movimiento impulsó y que el gobierno transformó en políticas de Estado. Un primer ejemplo de ello es 'Sin potreros no hay Maradonas', un programa de fomento a la construcción de canchas de fútbol barriales que el Evita comenzó impulsando en sus barrios de manera autogestiva y que luego se transformó en una política asumida desde el Estado:

"Nosotros todo el año pasado planteamos el eje 'Sin potreros no hay Maradonas'. Fuimos a manguearle pelotas a la subsecretaria de juventud (...) y nos dijeron: -'de esto queremos llenar el Estado, hagamos un programa, estructuremos esto como un programa nacional, laburémoslo juntos'. Era un eje de militancia nuestro (...) eso es un ejemplo de cómo nosotros generamos demanda, generamos organización, y después el Estado da respuesta” (Joaquín Noya-ME-. Concejal en el municipio de Vicente López).

El eje de los potreros en barrios populares en realidad formó parte de una propuesta más amplia que desde la JP Evita denominaron 'Paritaria Social Juvenil'. Esta 'paritaria' fue lanzada por el movimiento hacia 2014 y buscó visibilizar las demandas más acuciantes de los y las jóvenes y traducirlas en políticas públicas. Tal como expresó en un texto público Diego Fernández Camillo, el responsable de la JP Evita de la ciudad de La Plata, la 'paritaria' focalizaba en 5 ejes: tierra y vivienda, deporte y cultura, trabajo, educación, y violencia institucional ${ }^{\circ}$.

Precisamente durante ese año la militancia de la JP Evita recibió con enorme agrado la noticia de que Cristina Fernández de Kirchner había lanzado un programa especialmente dedicado a la juventud: PROGRESAR. Este implica un estipendio económico mensual a jóvenes entre 18 y 24 años que se encuentran desocupados o que cobran un sueldo menor al salario mínimo. La contraprestación que exige el Estado es que los/as jóvenes se formen en alguna institución educativa. Los militantes del Evita plantean que el espíritu de PROGRESAR recupera algunas de las líneas que estaban planteadas en la 'Paritaria Social Juvenil':

6 Más información en la página de Info Blanco sobre Negro de Buenos Aires: http://infoblancosobrenegro. com/noticias/7208-los-jovenes-del-movimiento-evita-quieren-construir-el-segundo-tomo-de-la-decadaganada 
"Cuando nosotros planteamos el tema de la paritaria social juvenil, son cosas que plantean los compañeros en los plenarios de la JP (...) nosotros lo habíamos pensado antes de que el gobierno nacional lo diga, varios meses. Lo veníamos charlando entre nosotros y cuando nos llaman a la Casa Rosada y escuchamos a Cristina que nos dice "el plan PROGRESAR" nosotros nos miramos y dijimos: -'esta es la asignación por estudiante que estamos hablando nosotros!'; entonces nos pusimos contentos" (Lucas -ME-).

Otra política pública impulsada desde el kirchnerismo tuvo que ver con el proyecto de ley aprobado en 2014 para que los jóvenes puedan votar en forma optativa desde los 16 años y no desde los 18 como preveía la ley anterior. En un texto titulado 'Vamos los pibes', uno de los referentes jóvenes del movimiento destacaba que la posibilidad de plantear ese debate tenía que ver con el ciclo kirchnerista en su conjunto:

“esta discusión es posible sólo en un proceso de restitución y conquista de derechos como el que venimos viviendo desde 2003 (...) sabemos, entonces, cual es la política de este proyecto para los jóvenes: más inclusión, más participación y más organización” (Grosso, 2012), dicha ley fue entendida entonces por el Movimiento Evita como un modo de "homenajear a los jóvenes militantes" (Telam, 2014).

Esta batería de medidas se dio desde la segunda presidencia de Cristina Fernández de Kirchner (2011-2014), ciclo en el cual "la juventud se convierte en una causa pública que promueve adhesiones y movilización” (Vázquez, 2013, p. 1). En ese contexto se fue instalando una narrativa acerca del 'regreso' de los jóvenes a la política. Marina Larrondo advirtió que el: “discurso kirchnerista hacia la Juventud (...) construye líneas de continuidades y rupturas en la historia de la Argentina reciente, y en particular a la “misión” de la juventud” (Larrondo, 2013, p. 359). Si coincidimos con Chorny en que durante este período se consolidó "un contexto de creciente protagonismo de la juventud como destinataria de políticas públicas” (2016, p. 208), entonces podemos pensar que dicho contexto ayudó a que la juventud del Evita se apropie del proyecto kirchnerista.

Un ejemplo de esta apropiación puede observarse en el 'juramento de lealtad a Néstor Kirchner' que la JP Evita organizó tras el repentino fallecimiento del ex mandatario en 2010. Cerca de 300 jóvenes del Evita declararon frente al mausoleo de Kirchner: “ ¿juramos sobre esta tumba defender el legado de lucha del pueblo argentino? ¿Juramos continuar el legado de Néstor para que de una vez y para siempre seamos un pueblo feliz en una Argentina liberada? Si, juramos" (Movimiento Evita, 2014). Para Joaquín ese juramento demuestra lo 
importante que es el kirchnerismo para el Movimiento Evita: "el juramento que le hizo la JP Evita a Néstor Kirchner es eso, nos identificamos como eso. El kirchnerismo para nosotros es una piedra fundamental. También somos hijos del kirchnerismo. El Evita no existía como existe hoy antes del kirchnerismo". (Joaquín Noya -ME-).

Para varios militantes entonces, las decisiones presidenciales le permitieron al Movimiento Evita crecer: "Leo es Diputado Nacional por todo lo que hacemos, pero también porque Cristina define. Y cuando Cristina definió fue cuando más espacios de poder tuvimos. Emilio es Secretario de Estado con Cristina, no en otro momento". (Joaquín Noya-ME-)․ Entonces, mientras que desde la JP se enfatiza en la externalidad del Estado y la autogestión de sus finanzas, también se celebra que el gobierno les haya otorgado lugares del poder dentro del Estado. Otro elemento sumamente valorado es que el gobierno haya tomado sus iniciativas para transformarlas en políticas de Estado o bien que haya impulsado políticas hacia la juventud en general.

El accionar del gobierno respecto de la juventud fue entonces positivo para la militancia de la JP Evita. Esto fue así en primer lugar porque se inscribió en la concepción general que tiene el movimiento acerca de integrar y solucionar el conflicto social desde el Estado. En segundo lugar, porque, como se vio a partir de los testimonios, entienden que la ampliación de derechos hacia la juventud fue posible porque se dio en el marco de un proyecto político más amplio que extendió derechos y recuperó la capacidad de gestión del Estado: ese proyecto es para ellos el que inició en 2003 de la mano de Néstor Kirchner. En tercer lugar, es probable que el énfasis en la discursividad kirchnerista, que destaca la bibliografía especializada, haya servido de estructura de oportunidades políticas para que la JP Evita reactualice la mística ligada a la transformación social a partir de la juventud.

\section{Conclusiones}

Los resultados expuestos permiten acceder a algunas conclusiones acerca del problema investigado. En primer lugar, en lo que refiere al lugar de la juventud en el movimiento las conclusiones reafirman que la mirada hegemónica reenvía a una noción asociada a valores positivos como la 'frescura', el 'empuje' y la 'vitalidad'. En el caso de algunos adultos estos valores incluyen, como con-

7 Se refiere a Leonardo Grosso, Diputado Nacional, y a Emilio Pérsico, cuyo cargo ya fue consignado. 
trapartida, una serie de 'carencias' que se asocian a la juventud, como la falta de capacitación y cierta ingenuidad política.

Pero en forma mayoritaria se reafirma entonces lo que varios estudios recientes planteaban acerca de que, en el plano discursivo, los militantes no dan cuenta de una disputa intergeneracional sino más bien de una confluencia de miradas en torno a que los jóvenes deben ir ganando cada vez más espacio en la organización. No obstante, en algunos adultos este discurso no responde tanto a un convencimiento sobre las virtudes de los jóvenes, sino más bien a una resignación sobre las debilidades propias.

Esto probablemente explique algunas realidades orgánicas internas del movimiento que fueron expuestas: se ha mostrado por caso cómo en un espacio relevante de toma de decisión como la Mesa Ejecutiva Nacional, la presencia de la juventud es escaza en relación a su enorme volumen de militancia. Y para matizar esto último, también es de notar cómo varios/as jóvenes fueron elegidos/as por el movimiento para acceder a importantes cargos legislativos.

En segundo lugar, acerca de la relación del movimiento con la gestión estatal, en términos generales se concluye en que el kirchnerismo fue leído desde el Movimiento Evita como un contexto en el cual se pudo integrar en forma virtuosa el conflicto social al plano de la institucionalidad estatal. Además, entienden que las decisiones presidenciales permitieron que el movimiento crezca y gane espacios en el Estado. Los resultados permiten concluir entonces que la adhesión de la militancia de la JP Evita al kirchnerismo tuvo varios fundamentos. En primer lugar, se ubican las políticas públicas destinadas a la juventud, como por ejemplo la oficialización del programa 'Sin potreros no hay Maradonas', el impulso al plan PROGRESAR o la ley de voto desde los 16 años. Complementariamente, esa adhesión también pudo responder a la apertura de la gestión kirchnerista hacia los movimientos sociales, entre ellos el Evita, lo cual significó que el movimiento colocase militantes en diversas escalas de gestión (nacional, provincial y municipal).

En lo que respecta a la relación directa de los jóvenes con la gestión estatal el panorama se muestra complejo y obliga a establecer matices; muchos/as jóvenes de la JP Evita remarcan y demuestran la externalidad de su militancia respecto de los recursos estatales. Esto lo hacen resaltando su rol de empleados en empresas privadas o de trabajadores/as independientes y la condición autogestiva de muchas de sus actividades.

Lo interesante aquí es que los jóvenes valoran la capacidad de gestión del Estado, pero no solamente cuando ellos mismos acceden a la gestión estatal, sino también cuando las demandas son integradas aún desde carteras de gestión a cargo de otros/as. Esto permite sugerir que la forma de apropiación y adhesión a un gobierno no solamente debe leerse en función de la integración directa que 
éste hace de los militantes de determinada organización, sino también de su capacidad de cumplir con sus expectativas político-ideológicas.

Lo anterior, no debe llevar a obviar, desde ya, el innegable relacionamiento de las actividades territoriales del Movimiento Evita con los recursos y programas estatales. Además, se ha mostrado cómo un porcentaje importante de jóvenes del Evita han ocupado lugares en las listas electorales del Frente Para la Victoria llegando, incluso, a acceder a cargos legislativos.

En suma, la juventud en el Movimiento Evita enfrenta un escenario en el cual se mixturan accesos y restricciones. Estas últimas representan una contradicción en relación con el enorme volumen de militancia que la juventud le aporta al movimiento. Aun así, la construcción de base y el acceso a cargos legislativos muestran que los y las jóvenes evidentemente vienen avanzando en crecimiento y organización. Este avance es visto con buenos ojos por la mayoría de los adultos, aunque algunos teman ser 'tirados por la ventana', por las nuevas y pujantes camadas militantes.

\section{Referencias}

Alcántara Sáez, M. (2008). La escala de la izquierda. La ubicación ideológica de presidentes y partidos de izquierda en América Latina. Nueva Sociedad, (217), 72-85.

Bourdieu, P. (1990). La juventud no es más que una palabra. En P. Bourdieu, Sociología y cultura (pp. 163-173). México: Grijalbo.

Boyanovsky, C. (2010). El Aluvión del piquete al gobierno. Movimientos Sociales y Kirchnerismo. Buenos Aires, Argentina: Ed. Sudamericana.

Cheresky, I. (2004). Elecciones fuera de lo común. Las presidenciales y legislativas nacionales del año 2003. En I. Cheresky \& J. M. Blanquer (Eds.). ¿Qué cambió en la política argentina? Elecciones, instituciones y ciudadanía en perspectiva comparada. (pp. 21-54). Rosario: Homo Sapiens.

Chorny, V. (2016). El discurso de los funcionarios sobre los jóvenes y las políticas públicas. Representaciones encontradas entre la Ciudad de Buenos Aires y la Provincia de Santa Fe. Argumentos, (18), 207-230.

Cortés, M. (2010). Movimientos sociales y Estado en el "kirchnerismo". Tradición, autonomía y conflicto. En A. Massetti, E. Villanueva \& M. Gómez 
(Comps.). Movilizaciones, protestas e identidades colectivas en la Argentina del bicentenario (pp. 97-118). Buenos Aires: Nueva Trilce.

Goldstone, J. (2003). States, Parties, and Social Movements. Cambridge: Imprenta de la Universidad de Cambridge.

Gómez, M. \& Massetti, A. (2009). Los movimientos sociales dicen. Conversaciones con dirigentes piqueteros. Buenos Aires, Argentina: Nueva Trilce.

Grosso, L. (octubre 11 de 2012). ¡Vamos los pibes! Movimiento Evita. Recuperado de https://movimiento-evita.org.ar/2012/10/11/\%C2\%A1vamos-los-pibes/

La Fogata (2003). Contra la farsa electoral por otro 20 de diciembre: que se vayan todos. Recuperado de http://www.lafogata.org.cn2.toservers.com/o03arg/ arg3/ar_farsa.htm

Larrondo, M. (2013). El discurso político kirchnerista hacia la juventud en contextos de actos de militancia. Astrolabio Nueva Época, (11), 334-363.

Longa, F. (2017). Cuando los movimientos llegan a la oficina. Dilemas del Movimiento Evita en un gobierno municipal de Buenos Aires, Argentina (20042015). (Con)textos: Revista D’antropologia i investigació, (7), 38-54.

Martín, E. (1998). Producir la juventud. Crítica de la sociología de la juventud. Madrid, España: Istmo.

Movimiento Evita (2014). Homenaje a Néstor Kirchner. Recuperado de https:// movimiento-evita.org.ar/2014/10/28/homenaje-a-nestor-kirchner-masde-300-jovenes-de-la-jp-evita-en-rio-gallegos/

Munck, G. (1995). Algunos problemas conceptuales en el estudio de los movimientos sociales. Revista Mexicana de Sociología, 57(3), 17-40.

Natalucci, A. (2012). Los movimentistas. Expectativas y desafíos del Movimiento Evita en el espacio kirchneristas (2003-2010). En G. Pérez, \& A. Natalucci (Eds.). Vamos las bandas. Organizaciones y militancia kirchneristas (pp. 27-56). Buenos Aires: Nueva trilce. 
Potter, J., \& Wetherell, M. (1987). Discourse and social psychology: Beyond attitudes and behaviour. Londres: Sage.

Quirós, J. (2008). Piqueteros y peronistas en la lucha del Gran Buenos Aires. Por una visión no instrumental de la política popular. Cuadernos de Antropología Social, (27), 113-131.

Sabino, C. (2000). El proceso de investigación. Buenos Aires, Argentina: Lumen.

Sherif, M., \& Sherif, C. W. (1975). Los problemas de la juventud en transición. En Sherif, M., \& Sherif, C. W. (Eds.). Problemas de la juventud actual. México D.F.: editorial Trillas.

Svampa, M. (2008). Cambio de época. Buenos Aires, Argentina: Siglo Veintiuno Editores.

Telam. Agencia Nacional de Noticias (2014). Podcast. Recuperado de http:// www.telam.com.ar/multimedia/audios/16456-leonardo-grosso-diputado-nacional-sobre-el-proyecto-de-ley-para-establecer-el-16-de-septiembre-como-dia-de-la-juventud-se-busca-homenajear-a-los-jovenes-militantes/

Unger, R. (1987). False Necessity. Anti-Necessitarian Social Theory in the Service of Radical Democracy. Nueva York: Imprenta de la Universidad de Cambridge.

Valles, M. (1997). Técnicas cualitativas de Intervención Social: Reflexión, metodología y práctica profesional. Madrid, España: Síntesis SA.

Vázquez, M. (2013). En torno a la construcción de la juventud como causa pública durante el kirchnerismo: principios de adhesión, participación y reconocimiento. Revista Argentina de estudios de juventud, (1), 1-25.

Vázquez, M. (2014). "Militar la gestión”: una aproximación a las relaciones entre activismo y trabajo en el Estado a partir de las gestiones de gobierno de Cristina Fernández de Kirchner en Argentina. Apuntes, (74), 71-102.

Vieytes, R. (2004). Metodología de la investigación en organizaciones, mercado y sociedad: epistemología y técnicas. Buenos Aires, Argentina: De las ciencias. 
Zarazaga, R. (2017). Introducción. El Estado Golem. En R. Zarazaga \& L. Ronconi (Comps.), Conurbano infinito. Actores políticos y sociales, entre la presencia estatal y la ilegalidad (pp. 9-18). Buenos Aires, Argentina: Siglo XXI.

Zibechi, R. (1999). La mirada horizontal: movimientos sociales y emancipación. Buenos Aires, Argentina: Editorial Nordan-Comunidad. 